\section{EL ANÁLISIS ORGANIZACIONAL COMO FUNDAMENTO PARA LA MEJORA DE} LA ESCUELA

\section{Isabel Pérez de Maldonado \\ Marisabel Maldonado \\ Suleima Bustamante Uzcátegui \\ UPEL-IPB.}

Núcleo de Investigación en Comportamiento Organizacional
Aceptado: 31-05-06

\section{Resumen}

El propósito de este artículo es dar a conocer el resultado de diversas experiencias, derivadas de procesos investigativos desarrollados en la Línea de Comportamiento Organizacional y Productividad, adscrita al Núcleo de Investigación en Comportamiento Organizacional de la UPEL-IPB. Estas se refieren al análisis realizado en instituciones educativas, como organizaciones, con el propósito de iniciar en ellas procesos de mejora. Se configura una estrategia de análisis, a partir de su aplicación en 12 instituciones, que plantea una revisión ecológica de la escuela; y examina, tanto el estado actual como el estado deseado de la organización, a partir de las expresiones de sus miembros, obtenidas mediante el empleo de diversas técnicas para la recolección de información. Una vez realizado el análisis de la información y en correspondencia con el estado deseado expresado, se fundamenta la elaboración de recomendaciones para la mejora de la dinámica organizativa institucional.

Palabras claves: Análisis organizacional, clima escolar y de aula, instituciones educativas.

\section{ORGANIZATIONAL ANALYSIS AS A MEAN TO IMPROVE SCHOOL}

\section{Abstract}

The purpose of this article is to show results of research experiences in the field of Organizational Behavior and Productivity from the Organizational Behavior of the UPEL-IPB Investigation and Research Nucleus. The goal of these experiences is to begin improving processes in educational institutes through analysis. This analysis implies an ecological revision of schools, and checks the current state and the desired state of the organization, taking into account their members' opinion, which has been collected through various techniques. Once the analysis is done, and considering the desired state of the organization, a set of recommendations is made to improve the organizational dynamics of schools.

Keywords: Organizational analysis, school and classroom organizational state, schools.

\section{INTRODUCCIÓN}

“...las buenas prácticas no son tales porque todo haya salido perfecto, sino porque han supuesto el inicio a un camino colectivo, dar la espalda a la vía de las lamentaciones para adentrarse en la senda de los proyectos positivos."

Muñoz-Repiso y Murillo

La presentación de estas experiencias investigativas se inicia con una caracterización de los elementos que intervienen en la dinámica organizacional, la cual se caracteriza en el nuevo contexto global, por los cambios acelerados de la ciencia y la tecnología y donde las organizaciones educativas son muy susceptibles de transformación. De modo que, las organizaciones se han concebido de diferentes maneras y desde diferentes perspectivas y ligada a esa concepción está la del hombre ejecutante del trabajo. Una organización, como lo señala Hall (1983), es

"una colectividad con unos límites relativamente identificables, un orden normativo, rasgos de autoridad, sistemas de comunicación y sistemas de pertenencia coordinado; esta colectividad existe de manera relativamente continua en un medio y se embarca en actividades que están relacionadas, por lo general, con un conjunto de objetivos”(p.33).

Más recientemente, Gairín (1999), expresa, 
“...hablar de organización supone considerar totalidades integradas por encima de totalidades aditivas; esto es, considerar realidades donde los elementos no son intercambiables entre sí, sino que mantienen una dependencia recíproca y adquieren sentido en función de un todo. Pero además, por su efecto autoevaluativo, aunque los procesos de organización vienen inducidos desde fuera, ...., pueden tener desarrollo propio a partir de procesos de autorregulación interna.”(p.77).

En estas definiciones se observa que las organizaciones como espacio social, ofrecen una asignación especial de valor a las personas y a los procesos que se derivan de la interacción de ellas, y de la calidad de los procesos también se deriva la calidad de los objetivos logrados en la organización, y en esos procesos de interacción se plantea la comunicación como medular en la dinámica organizacional. La comunicación en este ámbito se refiere a actos comunicativos ligados a la realización de actividades, pues trabajar juntos en las condiciones actuales de producción, es comunicar, en el sentido de construir y desarrollar un espacio de intersubjetividad y de comprensión recíproca.

En este orden, Flores (1997) destaca que, cuando en una organización los intercambios comunicativos se transforman en el núcleo central del proceso de trabajo, la organización se constituye en una red de conversaciones, lo que asigna al lenguaje un nuevo papel, que implica su dimensión instrumental, cognitiva y social. Así, la comunicación esencial en el proceso de cambio, es aquella generada desde la acción y que a su vez es generadora de acción.

Desde esta perspectiva, este artículo considera sucintamente las diversas concepciones de la organización y su evolución, como una forma para abordar con mayor precisión el estudio de los Instituciones Educativas. Así también se hace una descripción de una estrategia de análisis organizacional, que incluye la evaluación del clima escolar y el clima de aula, los cuales proporcionan información útil para la mejora de la dinámica organizativa en las instituciones

\section{LA CONCEPCIÓN DE ORGANIZACIÓN. ALGUNAS PRECISIONES SOBRE SU EVOLUCION}

Se discuten en este apartado tres concepciones de organización que han evolucionado en el tiempo. Una concepción plantea la organización como máquinas, según la cual las organizaciones se perciben como sistemas mecánicos, sin propósito propio, enmarcadas en una función mecanicista, pues, al igual que todas las máquinas, se creía que tenían la misión de servir al propósito de sus dueños; este era obtener un retorno adecuado sobre la inversión de tiempo, esfuerzo y dinero. Los patronos en estas organizaciones consideraban irrelevantes los propósitos e intereses del personal. Se retenían a los trabajadores sólo mientras hicieran lo que los dueños requerían. Cuando ya no era así, eran como piezas reemplazables de máquinas, desechados y sustituidos por otros que fueran dóciles y usables. Esto era así inclusive para los gerentes.

En los términos descritos previamente, se evidencia que las organizaciones conceptualizadas como máquinas se presentan como un sistema accionado desde fuera, controlado por otros; siendo así, es poco lo que las personas que la forman pueden hacer para modificar las condiciones existentes. No se abre espacio para la innovación, lo que pasa a ser un privilegio de quienes dirigen (Carrillo, 1997 a,b).

Otra concepción es la que se conoce como sistema biológico, el cual mantiene un sólo propósito, el de la sobrevivencia. Un sistema mantiene este propósito, trabajar sólo para la sobrevivencia, porque cree que esa es la manera de progresar. De acuerdo con Ackoff (1981), al final de la I Guerra Mundial, la concepción mecanicista fue reemplazada en gran parte por una concepción biológica; las organizaciones fueron vistas cada vez más como organismos que como máquinas. La razón más importante para la transición fue que sus dueños no podían explotar todas las oportunidades de crecimiento de sus organizaciones aún si reinvirtieran todas sus utilidades. 
Tuvieron que vender acciones, volverse empresas públicas y contratar gerentes profesionales. Las utilidades se vieron como un medio necesario para la supervivencia y crecimiento de la organización y no como su razón de ser.

De la concepción biológica surgió el nombre de corporación, una noción eminentemente biológica que significa cuerpo. Los trabajadores son tratados más como órganos difíciles de reemplazar que como piezas de máquinas fácilmente sustituibles; por consiguiente, la salud y la seguridad del personal se convirtieron en tema importante de discusión en la organización.

Una tercera concepción presenta a la organización como un sistema social. Esta, no solamente tiene propósitos propios, sino que se compone de otros sistemas menores que a su vez tienen sus propios propósitos. Ackoff señala que las organizaciones como sistemas sociales están orientadas hacia el desarrollo y no hacia el crecimiento. Expresa también este autor, que las funciones sociales de tales organizaciones son producir y distribuir riquezas; lo cual es realizable de distintas maneras, por ejemplo, compensando a empleados por su trabajo, pagando a los proveedores por sus bienes y servicios, proporcionando dividendos a los accionistas y pagando impuestos e intereses por el dinero prestado. La creación de empleo productivo se establece como de vital importancia en las organizaciones que funcionan como sistemas sociales.

La concepción de una organización se refleja en sus temas principales. Mientras que en las organizaciones como sistemas mecánicos y también biológicos los temas son economía y tecnología, en la organización como sistema social hay dos temas adicionales: ética y estética. En el primer caso hay información y conocimientos, mientras que en el segundo, hay además, comprensión y sabiduría (Inda, 1997). Estos dos últimos temas contribuyen al progreso humano de la organización, por cuanto su esencia está en la inserción de valores en la toma consciente de decisiones.
Una organización, para que funcione como sistema social también debe cumplir, según Ackoff (1981) con otro requisito, esto es, ser sensible a las demandas del ambiente interno y externo a ella, y en consecuencia, responder con rapidez y eficacia a tales demandas. Esta respuesta y su calidad dependerán de los métodos que emplee para abordar la situación detectada.

Anteriormente se pensaba que había maneras específicas, concretas y en muchos casos una única manera de resolver un problema, de fundamentar las decisiones. Y eso fue cierto y dio resultado. Fue el abordaje de la vida empleado por el mundo Occidental; se trataba entonces de un enfoque analítico, donde cada problema se fragmentaba, bajo el concepto de que estudiando cada parte se conocía la totalidad.

Hoy día se impone en primer lugar, creer que existen maneras diferentes de abordar cada asunto y en segundo lugar, que en vez de emplear exclusivamente el enfoque analítico, es necesario manejar el enfoque sistémico, que consiste en estudiar la totalidad considerando las interacciones de las partes, empleado en el mundo Oriental.

Para el abordaje de los problemas a nivel organizacional incluyendo el enfoque sistémico, se requiere que los gerentes se actualicen constantemente, aproximándose al cúmulo de conocimientos que los potencie para realizar una acción gerencial acorde con las exigencias actuales. Por cuanto, durante miles de años, la información acumulada por la humanidad creció a un ritmo lento, casi imperceptible. No obstante, en los últimos siglos, el volumen de conocimientos se incrementa progresivamente, comenzando una curva de despegue con la revolución industrial. Algunas estimaciones actuales calculan que en un campo como en la ingeniería informática la cantidad de información disponible se duplica cada cinco años; en el nuevo siglo se duplicará cada año (Bartolomé, 1997).

Cabría entonces preguntarse, a partir de esas concepciones, verdaderamente habrá cambiado en el tiempo, en la práctica la manera de 
concebir la organización y esencialmente la forma de observar a las personas que las constituyen? . Desafortunadamente la respuesta es negativa, pues nuestra experiencia investigativa nos lleva a señalar que la concepción de la organización no es un asunto que se documenta solamente sino que quienes dirigen la organización deben experimentarlo. De aquí que al momento de estudiar cualquier organización sea necesario conocer cómo se considera esta. Es decir, cómo es vista: un sistema mecánico, un sistema biológico o un sistema social?; pues sabemos que esto nos permitirá saber la concepción que se tiene del ser humano que desarrolla actividades en la organización.

Con base a estas apreciaciones, la figura 1 permite visualizar a la organización como sistema social que está caracterizado por la dinámica interrelacional de factores humanos y técnicos.

Ver la organización escolar, la institución educativa o la escuela, como un sistema social, implica entonces, reconocerla como la agrupación de profesores, estudiantes y miembros de la comunidad en general (personal de apoyo, padres y representantes, ...) que poseen características propias y exige asignarle tanta importancia a la consideración de los elementos que la integran como a sus interrelaciones intrínsecas y extrínsecas. Desde esta concepción, en la institución educativa se distinguen varias dimensiones de análisis; como señala Gairín (1999), “... la organización escolar como un factor conglomerante de contextos generales (lo socio-cultural, lo educativo y lo escolar) y específicos y, a su vez, como un sistema en sí misma, no podemos extrañar que califiquemos su realidad como compleja”.(p.63)

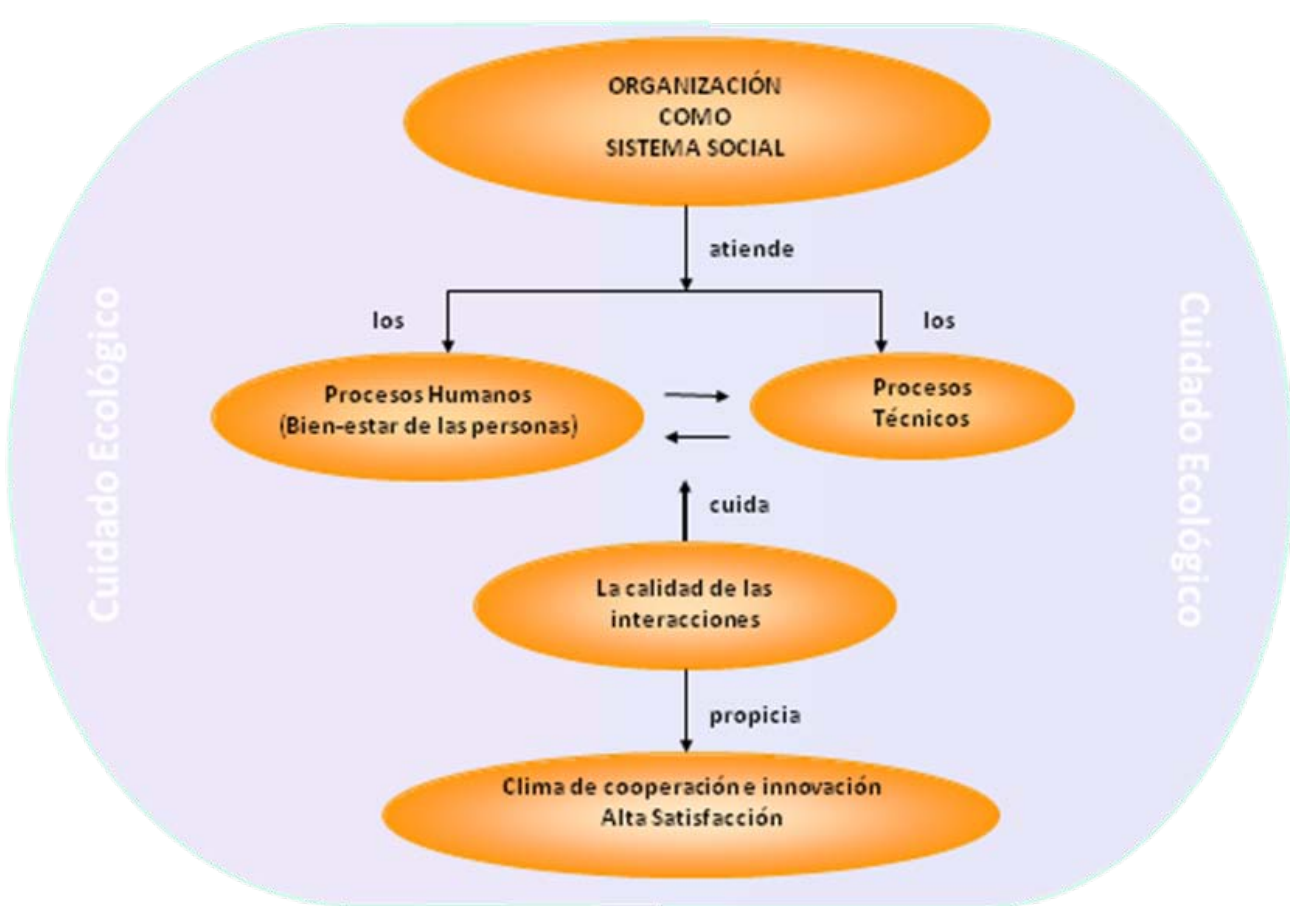

Figura 1. Organización como sistema social. (Pérez de M., 2000)

La institución educativa como unidad de análisis, remite a la consideración de sus dimensiones, las que a nuestro juicio orientan el proceso investigativo. Así podemos mencionar, siguiendo el planteamiento de Frigerio y otros (1992), que las dimensiones de análisis se refieren a: lo organizacional, lo pedagógico-didáctico, lo comunitario y lo administrativo. Es oportuno señalar, que el análisis de las instituciones educativas referidas en este artículo, se ocupa de la dimensión organizacional, por cuanto consideramos que es esta dimensión, su dinámica (que en adelante la señalamos como dinámica organizativa) lo que posibilita u obstaculiza en gran medida una acción educativa exitosa. La dinámica organizativa de la escuela se caracteriza por mantener una estructura de valores, roles y normas, que se relaciona en gran medida con el contexto socio-cultural que privilegia su entorno. De esta manera, al pretender abordar la escuela como 
organización es indispensable identificar esos tres componentes, por cuanto son ellos los que orientan la acción organizacional.

$\mathrm{Al}$ referirnos a la estructura de roles y a la existencia de ciertas normas presuponemos la existencia de un modo organizacional particular de relacionarse las personas en la organización y con los procesos de trabajo, desde un marco regulativo, y los valores actúan como prescriptores de la acción. En este sentido, iniciar el análisis de la escuela como organización, con la caracterización de esos tres componentes valores, roles y normas, permitirá conocer si atiende a las condiciones que se establecen para una organización como sistema social.

\section{DESCRIPCIÓN DE LA ESTRATEGIA DE ANÁLISIS ORGANIZACIONAL}

Las investigadoras hemos considerado conveniente abordar el estudio de las instituciones educativas, mediante una estrategia de análisis organizacional, que incluya la evaluación del clima escolar y el clima de aula. Por cuanto la experiencia investigativa llevada a cabo permitió la obtención de resultados que proporcionan información de utilidad para la mejora de la organización, especialmente, la escuela.

La literatura que reporta prácticas de mejora escolar, presenta una variada gama de estrategias para analizar las instituciones educativas, desde la dimensión organizacional. Ellas coinciden en su finalidad, proporcionar información útil a la gerencia de las organizaciones de modo que a partir de ellas, es como podrán diseñarse acciones y luego lograr la mejora organizacional. (González R., 2000; Brighouse y Woods,2.001; Uría, 2001; Murillo y Muñoz-Repiso, 2002 ).

En este sentido se considera apropiado describir el trabajo realizado por Pérez de M.(2.000), que consiste en una propuesta de revisión ecológica, diseñada para analizar una organización; en la cual plantea la revisión del estado actual y del estado deseado. En el abordaje del estado actual, se considera a la organización como un todo, y se revisa tanto su contexto, como lo organizativo, tomando como centro a las personas. En el contexto de la organización, revisa lo medular, como lo son sus principios filosóficos, por ser estos aspectos culturales de indudable efecto en la acción.

En el abordaje de lo organizativo, Pérez considera al clima como indicador de la calidad de las interacciones, y la satisfacción en el trabajo como expresión afectiva hacia éste. También contempla como parte de la revisión del estado deseado, la expresión por parte de los miembros, de cómo desean que sea la organización y cuáles acciones, proponen realizar para lograr el estado deseado. La revisión ecológica fue aplicada en una empresa, y sus resultados, en opinión de los gerentes de la empresa, fueron útiles para diseñar mejoras organizacionales.

El enfoque ecológico planteado por Pérez de M (ob.cit.), contempla el proceso de elegir adecuadamente los cambios que se van a realizar, así como las estrategias y las actividades a poner en práctica, a fin de lograr en el personal la mejora deseada, al tiempo que se cuida su bienestar. Desde esta perspectiva, la ecología de las organizaciones plantea que estas y sus ambientes de trabajo, están comprometidos en un patrón de cocreación, donde cada uno produce y potencia al otro. Vistas así, las organizaciones tienen un rol activo en la delineación de un futuro exitoso, especialmente cuando actúan concertadamente atendiendo a su clima.

En particular, en las organizaciones educativas las estrategias que se empleen para su análisis, deberían, como apunta Gairín (1999), integrar lo didáctico y lo organizativo. En atención a ello, la revisión ecológica presentada por Pérez de M (2.000), fue adaptada al ámbito educativo y aplicada y modificada a través de su aplicación en varios centros educativos (en aproximadamente 12 centros). Algunos de los resultados obtenidos se reportan en Pérez de M y Maldonado (2004ayb); Pérez de M, Maldonado y Bustamante (2004); Pérez de M, Maldonado y Bravo (2004).

La estrategia, como lo muestra la figura 2, consiste en una revisión ecológica, que incluye dos aspectos. El estado actual de la organización y el estado deseado. En la revisión del estado actual se examina el contexto 
organizacional, mediante la cual se obtendrá información suficiente para conceptualizar el sistema; se busca detectar, en cuanto se aproxima a la conceptualización de la organización como sistema social; se indaga sobre los temas principales que interesan al centro educativo, como los principios rectores, trabajo cooperativo, toma de decisiones, aspectos organizacionales que permitan detectar si existe coherencia entre el Proyecto Educativo, sus principios rectores, el aprendizaje cooperativo, y el conocimiento y la actuación de la comunidad, la participación de los actores de la comunidad en la toma de decisiones, entre otros. Esta información se obtiene mediante entrevistas y sesiones de trabajo con el personal directivo del centro.

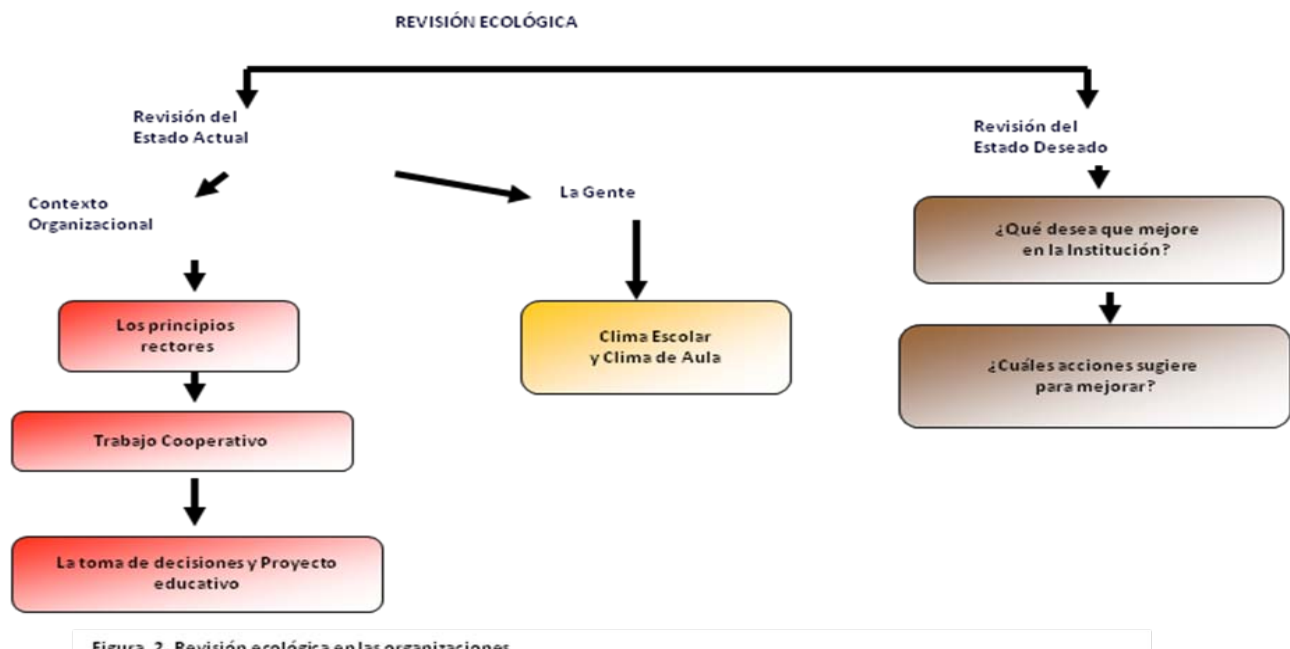

\section{Figura 2. Revisión ecológica en las organizaciones}

El otro punto que se examina en la revisión del estado actual, es lo organizativo centrada en la gente, se estudia el clima escolar, específicamente el clima organizativo o institucional, y el clima de aula. Se busca conocer lo que piensan y sienten sobre su realidad organizacional y lo que desean se mejore en el centro educativo. El clima escolar es una dimensión bien diferenciada de la realidad escolar, que es digno de ser analizado, por cuanto es un facilitador/obstaculizador de los resultados, en la medida que contribuya efectivamente a los logros del centro. Para su análisis y valoración, se consideran los siguientes aspectos humanos fundamentales: (a)las relaciones humanas de todos los estamentos del Centro, entre sí y con los demás; (b)el orden y respeto mutuos, que hagan posible la maximización del tiempo útil de aprendizaje; (c)el sistema disciplinar: su elaboración más o menos participativa y su aplicación coherente; (d)el ejercicio pedagógico del tratamiento de la indisciplina; (e)el tipo de relaciones entre el personal: competitivas o individualistas; (f)Los valores efectivamente vividos en el centro; (g)el clima de expectativas elevadas de cara a los niveles personalizados de rendimiento; (h)la confianza en el éxito y (i)la satisfacción por el propio trabajo. Para realizar esta revisión se procede a aplicar los cuestionarios para evaluar el clima organizacional y la satisfacción en el trabajo, así como obtener las mejoras sugeridas.

Por último la revisión exige conocer el estado deseado para la organización. Esta revisión parte de los resultados obtenidos en la revisión del estado actual y será guiada por las preguntas contenidas en el diagrama, a fin de preparar el plan de mejora de la organización, teniendo un cuidado ecológico.

Esta estrategia de análisis ha sido empleada para estudiar al menos 12 centros educativos y la experiencia acumulada hasta la fecha permite afirmar que es útil para identificar aspectos necesarios para mejorar el centro a partir del sentir y pensar de los miembros de la organización.

\section{¿CÓMO SE OBTIENE LA INFORMACIÓN?}

Para obtener la información requerida, se aplican varias técnicas de recolección. Entre ellas: entrevista semi-estructuradas, grupos focales, 
cuestionarios. Específicamente, se emplea la entrevista semi-estructurada para obtener la información respecto al contexto organizacional.

Los cuestionarios para obtener información sobre el clima escolar y de aula, por parte de profesores y estudiantes; estos cuestionarios son varios: CLIOR para evaluar el clima escolar en los profesores y SES, para evaluar el clima en estudiantes. El clima escolar se define como la percepción que tienen de los docentes y estudiantes de la resultante de la interacción de los diferentes componentes organizativos, que son específicos en cada centro. CLIOR, mide la percepción de los docentes sobre la calidad del ambiente psico-social-laboral en las instituciones seleccionadas para la investigación; se refiere por lo tanto al clima organizativo percibido en los centros. Este instrumento es una adaptación del presentado por Álvarez (1992), consta de 55 ítemes, distribuidos en las dimensiones de respeto, confianza, estímulo, participación, crecimiento, apertura, comunicación, toma de decisiones, atención e identificación. La escala de respuesta empleada es de 6 puntos, variando desde completo desacuerdo (1) hasta completo acuerdo (6). Su validez fue evidenciada mediante el juicio de expertos y su confiabilidad se obtuvo mediante el Alpha de Cronbach, obteniéndose 0,91 para el instrumento en su totalidad. Resultados de la aplicación del instrumento CLIOR se presentan en Pérez de M y Maldonado (2004a).

El cuestionario SES, para medir Clima Escolar, a partir de las opiniones de los estudiantes, orientado en cuatro contextos, Interpersonal, Instruccional, Regulativo e Imaginativo; se asume que estos cuatro contextos conforman el clima escolar. Esta escala fue diseñada en1980 por Kevin Marjoribanks, en la universidad de Adelaida, Australia, y adaptado al contexto español por Villa Sánchez (1992). Consta de 28 ítemes, con una escala de respuesta de seis categorías. La escala de respuesta empleada es de 6 puntos, variando desde completo desacuerdo (1) hasta completo acuerdo (6). Resultados de la aplicación del instrumento SES se presentan en Pérez de M., Maldonado y Bravo (2004).

En cuanto al clima de aula, se examina la opinión de cada docente, en correspondencia con la opinión de su grupo de estudiantes. Para recolectar la información, se emplearon dos cuestionarios de preguntas abiertas. Para obtener la información requerida en este estudio, se diseñaron dos cuestionarios de preguntas abiertas, con el propósito de obtener información, lo más amplia posible, en los propios términos del encuestado. Las preguntas se refieren a lo siguiente: (a) tipos de problemas o conflictos que se presentan en el aula; (b) cuándo suceden la mayoría de las situaciones de conflictos?; (c) quienes son los causantes de los conflictos; (d) en qué medida afectan los conflictos en la clase, los resultados académicos; (e) qué se ha hecho para resolver los conflictos en el aula; (f) durante este lapso mejoraron la convivencia y los comportamientos en el aula; (g) qué piensan los docentes se podría hacer para mejorar el clima en el aula y los comportamientos del grupo. Los cuestionarios se aplicaron en forma colectiva. En el caso de los estudiantes, se aplicó el cuestionario en el aula; y en el de los docentes en conjunto, por institución.

También se emplea el cuestionario ACOP, con el cual se recoge información sobre aspectos relacionados con las experiencias estudiantiles. Este es un cuestionario conformado por preguntas abiertas y preguntas cerradas. Más información sobre este cuestionario y resultados se presentan en Pérez de M., Maldonado y Bravo (2004; 2006).

Los grupos focales y las entrevistas grupales se realizan para indagar sobre el clima escolar y posibilidades de mejora del clima en docentes, estudiantes, y miembros de la comunidad educativa en general, que incluye a padres y representantes. El protocolo para los grupos focales y para la entrevista grupal, se elabora después de realizar una revisión preliminar de la información que se obtenga de la aplicación de los instrumentos descritos con anterioridad.

Finalmente se busca información sobre las mejoras sugeridas. Se refiere a los aspectos que el personal docente, directivo y estudiantes de las instituciones educativas desea que se mejoren para el bienestar de todos, tanto en el área humana, como en el proceso de trabajo. Para obtener esta información se emplea un instrumento tipo formato que consta de una 
pregunta abierta en la que se solicita a los entrevistados que expresen: qué deseas que se mejore en la institución y qué acciones sugieres?.

\section{APLICACIÓn DE LA ESTRATEgIa DE ANÁLISIS ORganizaCIONAL. AlgunOS RESULTADOS OBTENIDOS.}

Los resultados del análisis del contexto revelan, ausencia y/o desconocimiento por parte de los directivos de los principios rectores institucionales, dificultad para llevarlos a la práctica, proceso no sistemático para la toma de decisiones, concepciones disímiles de lo que implica el trabajo cooperativo, entre otros. Se observó, discrepancia entre directivos de la misma institución, en cuanto a cuáles son los principios rectores de la institución, visión, misión y valores; así mismo, cual es el Proyecto Educativo que los integra como una comunidad. Es importante señalar que cuando se les pidió que señalaran concretamente algunos de los valores que se promueven en la escuela, en la mayoría de los casos, no se obtuvo información.

La toma de decisiones, señala la mayoría de los directivos, se realiza en equipo o como también expresan, en forma colegiada, incorporando siempre a las personas involucradas; en algunos casos, expresan, se reúnen primero los directivos y luego llevan planteamientos concretos al consejo de profesores y allí toman la decisión. En cuanto al aprendizaje cooperativo, se observó un manejo inadecuado del significado, alcance y ámbitos de aplicación; se hace necesario dar a conocer a los directivos sobre este tema por cuanto esto es crucial para mejorar la convivencia en los centros educativos y mejorar la calidad de la docencia.

Se les preguntó sobre la relación de la escuela con la comunidad educativa. Además de la discrepancia apuntada antes entre las expresiones de los directivos, se observó una relación poco enriquecedora; por cuanto al parecer sólo intervienen en el momento en que se necesita solventar algún problema o se necesitan recoger fondos para realizar alguna actividad.
Es evidente, que cuando el personal directivo no comparte los principios que rigen la organización, cuando no hay claridad en el proyecto educativo que los integra, por decir lo menos, es difícil esperar que la toma de decisiones, la participación de los actores y el logro de las metas educativas se conduzcan hacia la excelencia; se requiere de la gerencia acciones consensuadas, las cuales deben partir de un conocimiento compartido de los principios rectores y del Proyecto Educativo. La gerencia debe establecer conexiones entre la acción docente (en el aula y fuera de ella) y la organización escolar como dos realidades claramente diferenciadas; cada una de ellas con identidad propia, a la vez que entrelazables, para que, como señalan algunos autores, se genere una relación mutua que enriquezca a ambas realidades y les proporcione una perspectiva nueva y más amplia. (Gonzáles R., 2000; Pérez de M, 2000; Brighouse y Woods,2.001; Uría, 2001; Pérez de M. y Maldonado, 2004a).

En cuanto al clima escolar expresado por los docentes se ha reportado en Pérez de M. y Maldonado (2004a), lo siguiente: (a) los docentes perciben que no tienen en la escuela un clima de confianza y respeto entre colegas, ni entre el personal docente y directivos; (b) escasa participación en la toma de decisiones; (c) la comunicación se percibe como una debilidad; entre otros resultados. Lo relevante en este artículo, es que la evaluación que se hace en cada escuela proyecte resultados diferentes, propios de esa organización y es a partir de esos resultados que se generan acciones para la mejora, en este caso, del clima escolar.

En cuanto al clima escolar visto por los estudiantes, se reportan algunos de los resultados obtenidos. La tabla 1 muestra las puntuaciones promedio asignadas por los estudiantes en cada contexto del clima escolar y la puntuación global. 
Tabla 1. Evaluación del Clima Escolar por parte de los estudiantes.

\begin{tabular}{|c|c|c|c|c|}
\hline $\begin{array}{l}\text { Contexto } \\
\text { Interpersonal }\end{array}$ & $\begin{array}{l}\text { Contexto } \\
\text { Regulativo }\end{array}$ & $\begin{array}{l}\text { Contexto } \\
\text { Instruccional }\end{array}$ & $\begin{array}{l}\text { Contexto } \\
\text { Imaginativo }\end{array}$ & $\begin{array}{l}\text { Clima } \\
\text { Escolar }\end{array}$ \\
\hline $\begin{array}{l}\text { Expresa la } \\
\text { percepción de } \\
\text { los alumnos, } \\
\text { con respecto a } \\
\text { la calidad } \\
\text { interpersonal, } \\
\text { de amistad y } \\
\text { confianza por } \\
\text { parte de sus } \\
\text { profesores }\end{array}$ & \begin{tabular}{ll}
\multicolumn{2}{|l}{ Expresa } \\
percepciones \\
de & los \\
alumnos & \\
sobre & el \\
afecto o la \\
severidad de \\
las relaciones \\
autoritarias \\
con los \\
profesores y \\
el ambiente
\end{tabular} & $\begin{array}{l}\text { Expresa las } \\
\text { percepciones } \\
\text { de los } \\
\text { alumnos } \\
\text { sobre la } \\
\text { orientación } \\
\text { académica, } \\
\text { con énfasis en } \\
\text { el interés o } \\
\text { desinterés } \\
\text { que tienen los } \\
\text { profesores por } \\
\text { el aprendizaje. }\end{array}$ & 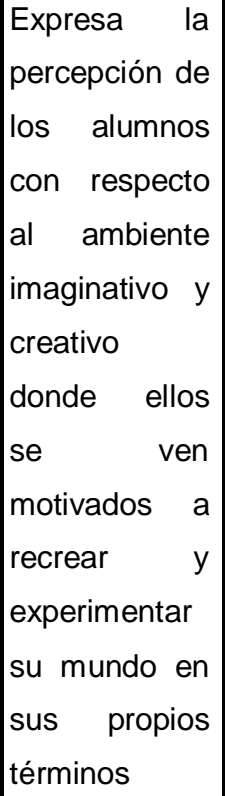 & $\begin{array}{l}\text { Percepción } \\
\text { que los } \\
\text { individuos } \\
\text { tienen de } \\
\text { los distintos } \\
\text { aspectos } \\
\text { del } \\
\text { ambiente } \\
\text { donde se } \\
\text { desarrollan } \\
\text { sus } \\
\text { actividades } \\
\text { habituales. }\end{array}$ \\
\hline 21 & 3.16 & 96 & 3.58 & 3.46 \\
\hline
\end{tabular}

Fuente: Pérez de M., Maldonado y Bravo, 2004.

Los resultados se interpretan en términos de Fortaleza, Oportunidades para mejorar y Debilidades sobre las cuales se requiere trabajar. Esta clasificación permite la discusión de los resultados con los miembros de la escuela a fin de comprender la percepción colectiva del clima escolar y derivar de allí acciones para la mejora.

\begin{tabular}{|l|l|}
\hline Puntuación & Categoría \\
\hline $5-6$ & Fortaleza \\
$<5$ hasta 4 & Oportunidad \\
$<4$ & Debilidad \\
\hline
\end{tabular}

Como se aprecia en la tabla 1, los estudiantes perciben el clima escolar como una debilidad, con lo cual y tomando el contenido de cada contexto se derivaron acciones concretas tanto a nivel de la escuela como globalidad como por parte de directivos y estudiantes.

Las vivencias estudiantiles asociadas a la participación de los estudiantes en la toma de decisiones, resultó escasa en cuanto al liceo y regular en cuanto al aula; se reporta la importancia dada en el liceo a las opiniones de los estudiantes como baja, y a la opinión de los padres y representantes se le ubica entre moderada y baja. Se concluye que es muy difícil mantener un clima favorable, si la institución no proporciona oportunidades a los estudiantes, para la participación y desarrollo de formas de convivencia, donde se sientan protagonistas de la vida en el liceo. Este estudio adquiere implicaciones relevantes al aportar información para profundizar en la importancia pedagógica y de gestión, lo cual ocurre cuando se generan en los centros educativos formas de convivencia más democráticas y participativas. (Pérez de M., Maldonado y Bravo, 2004)

Por otra parte, la evaluación del clima de convivencia en las aulas fue reportada por Pérez de M., Maldonado y Bustamante (2004). Los docentes y estudiantes coinciden en: (a)los conflictos ocurren en el aula como consecuencia de indisciplina, cuando trabajan en grupo y durante los recesos; (b) los alumnos son los generadores de los conflictos. Los docentes señalan, además, que los conflictos se producen por lo siguiente: falta de atención de los alumnos, carencia de hábitos de estudio y falta de orientación de sus representantes; los cuales inciden en los resultados 
académicos. Los docentes y estudiantes reconocen, que después de aplicar algunas estrategias de aprendizaje, por iniciativa del propio docente, mejoraron de manera importante la convivencia en el aula y los comportamientos. Se concluye señalando la necesidad de mantener en el aula climas afectivos, enriquecedores y niveles reales de aceptación del otro, con la finalidad de poder contribuir al desarrollo de personas autónomas, capaces de entender, a la vez, la autonomía y la dependencia de los demás.

Es importante señalar que al momento de preparar este artículo no se había analizado la información proveniente de otros miembros de la comunidad educativa, como lo constituyen el personal de apoyo de las instituciones y padres y representantes. Más información sobre la consulta a estos miembros de la comunidad se describe en Pérez de M, Maldonado y Bravo (2006)

\section{¿QUÉ APORTA ESTA EXPERIENCIA INVESTIGATIVA?}

Los procesos de investigación desarrollados con el propósito de realizar un análisis organizacional en las instituciones educativas, mediante la aplicación de la estrategia descrita en este trabajo, nos permite presentar algunas precisiones y o aportes, entre ellos:

1. La aplicación de la estrategia de análisis organizacional permite apreciar la dinámica de la escuela, a partir de lo que los miembros de la organización piensan y sienten.

2. Conocer lo que los miembros de la organización expresan respecto tanto al contexto organizacional, como al clima escolar y de aula, pues permite identificar necesidades de mejora en la escuela. De igual modo, la información obtenida cuando se les pregunta cómo quieren que sea y se den las cosas en la escuela, constituye un insumo fundamental para desarrollar planes de acción dirigidos a lograr la mejora deseada.

3. La organización de los planes de mejora de la escuela, estableciendo prioridades, llevan a construir un futuro deseable como posible, con lo cual se desarrolla entre los miembros de la organización un mayor compromiso.
4. La aplicación de la estrategia para el análisis de la organización no tendrá ninguna utilidad si la información que se obtiene no se traduce en planes para la mejora institucional, mediante el consenso de los miembros.

5. Es necesario que el equipo directivo, como dinamizador de la vida de la escuela, desarrolle un clima que propicie la reflexión permanente y compartida sobre la acción educativa que se realiza en la misma la escuela; un clima que promueva la reflexión, la creatividad, la innovación, que genere un mayor compromiso con el Proyecto Educativo de la escuela, la realización de encuentros entre los docentes y personal directivo, para reflexionar y diseñar acciones conjuntas, a fin de mejorar el ambiente de trabajo.

6. La aplicación de la estrategia de análisis abarca la indagación en las aulas. Brinda información útil para proporcionar a los docentes y estudiantes la oportunidad de crear un entorno seguro y ordenado que ofrezca a todo el alumnado la posibilidad de participar en un clima con multiplicidad de interacciones que fomenten la cooperación y la cohesión del grupo. Unas interacciones presididas por el afecto que contemplen la posibilidad de equivocarse y realizar las modificaciones oportunas, donde convivan la exigencia de trabajar y la responsabilidad de llevar a cabo el trabajo autónomamente, la emulación y el compañerismo, la solidaridad y el esfuerzo; unas interacciones que generen sentimientos de seguridad y contribuyan a formar en el alumno una percepción positiva y ajustada de sí mismo.

7. Encontramos que es indispensable cuidar el clima escolar y de aula, como una condición esencial para lograr la convivencia armónica y productiva en la escuela. De allí que compartamos los principios siguientes:

a) La Tarea de la Educación Escolar, como espacio de convivencia, es permitir y facilitar el crecimiento de niños y jóvenes como seres humanos para que actúen con responsabilidad y libertad en la comunidad a la cual pertenece.

b) La Educación debe ser un proceso de Transformación en la convivencia, en el que los niños y jóvenes se transforman en su vivir, 
de manera coherente con el vivir del profesor.

c) La convivencia escolar constituye, unos de los temas básicos en la pedagogía. El aprendizaje como proceso por el cual una persona adquiere o desarrolla una nueva conciencia y conocimiento, le proporciona nuevos significados al aprendiz.

d) El aula constituye un espacio institucional privilegiado para construir la convivencia en la escuela. El trabajo en grupo y el trabajo cooperativo, así como la mediación, constituyen técnicas efectivas para el aprendizaje de la convivencia.

e) La actitud y el compromiso de los directivos de la organización escolar es determinante para lograr el éxito en los proceso de construcción de la convivencia.

f) Todos los actores institucionales son responsables del modo de convivencia; no obstante, la mayor responsabilidad siempre recae en los adultos.

g) La organización escolar debe crear las condiciones que permitan a los niños y a los jóvenes, ampliar su capacidad de acción y reflexión sobre el mundo en que viven, de modo que puedan contribuir a la transformación y evolución de este.

\section{REFERENCIAS}

Ackoff, R. (1981). Creating the Corporate Future. Capítulo 1. "our Changing Concept of the world”. New York: John Wiley \& Sons.

Alvarez, G. (1992). El Clima Organizacional en instituciones educativas: Conceptualización, Investigaciones y resultados. Revista Interamericana de Psicología Ocupacional. Vol. 11, No 1y 2..
Bartolomé P., A. (1997). Preparando para un nuevo modo de conocer. Departamento de Didáctica y Organización Educativa. Universidad de Barcelona. Internet: España.

Brighouse, T y Woods, D. (2.001). Cómo mejorar los Centros Docentes. España: Editorial La Muralla.

Carrillo P., R. (1997a). La Gerencia de la Gente. Revista Calidad Empresarial. Innovación. Productividad. Competitividad. $\mathrm{N}^{0} 2$, enerofebrero.

Carrillo P., R. (1997b). El problema no es de recursos humanos, es cómo lo gerenciamos. Revista Calidad Empresarial. Innovación. Productividad. Competitividad. No5.

Flores, F. (1997). Creando organizaciones para el futuro. Chile: Dolmen ediciones Granica

Gairín S., Joaquín. (1999). La organización Escolar: contexto y texto de actuación. Madrid: La Muralla, S.A.

Gonzalez R., T (2.000). Evaluación y Gestión de la Calidad Educativa.. Un enfoque metodológico. Málaga, Ediciones Aljibe, S.L.

Hall, R. (1983). Organizaciones: estructura y procesos. Nueva York: Prentice-Hall.

Inda, A. (1997). El Mapa. Una guía para el mejoramiento de la calidad en la pequena y mediana empresa, basada en el método de W. Edwards Deming. USA: OEA.

Murillo, F. y Muñoz-Repiso, M. (2002). La mejora de la escuela. Un cambio de mirada. España: Octaedro. 
Pérez de Maldonado, I. (2000). Modelo de Acción Pedagógica para Capacitar a Gerentes en las Empresas. Medellín, Colombia. No2, pp. 6779

Pérez de Maldonado, I y Maldonado, M. (2004a) Análisis Organizacional en Instituciones Educativas. Revista Encuentro Educacional. Maracaibo, LUZ, Facultad de Humanidades y Educación. Vol. 11, pp.448-459.

Pérez de Maldonado, I y Maldonado, M. (2004 b). La Convivencia en las Aulas. LIV Convención Anual de Asovac. Acta Científica Venezolana: 55(sup.1) p. 529

Pérez de Maldonado, I , Maldonado, M. y Bustamante, S. (2004). La Convivencia en el aula como factor clave de Aprendizaje. Revista Laurus. UPEL. Año 10 N 18. pp 69-82.

Pérez de Maldonado, I , Maldonado, M. y Bravo, U. (2004). Clima Escolar y Vivencias Estudiantiles. LIV Convención Anual de Asovac. Acta Científica Venezolana: 55(sup.1) p. 533.

Pérez de Maldonado I., Maldonado M. y Bravo U. (2006). Fortalecimiento del clima de convivencia y mejoramiento de indicadores de calidad: Una Propuesta. Revista Laurus, UPEL, Año 12 N 21, pp.70-85.

Uría, M. (2.001) Estrategias Didáctico Organizativas para Mejorar los Centros Educativos. Madrid: Narcea S.A. de Ediciones.

Villa Sanchez (1992) en Villa S., A. y Villar A., L. (1992). Clima Organizativo y de Aula. Teorías, Modelos e Instrumentos de Medida. España: Publicaciones del Gobierno Vasco. 